\title{
PARKINSON DISEASE
}

\section{Disease-linked morphometric variants of $\alpha$-synuclein}

Two recent studies demonstrate that variants of $\alpha$-synuclein have different contributions to pathophysiology of disorders such as Parkinson disease (PD) and dementia with Lewy bodies.

Peelaerts and co-workers explored the pathophysiological effects of different conformations of $\alpha$-synuclein by injecting purified oligomers, ribbons and fibrils of human a-synuclein into the substantia nigra of rats.

7 days later, all three forms of the protein were taken up by dopaminergic neurons, but oligomers spread most widely throughout the brain. After 4 months, rats injected with ribbons or fibrils, but not with oligomers, showed neuropathology similar to Lewy bodies. Ribbons produced the highest burden of inclusion body pathology, but fibrils caused the most cell death in the substantia nigra, which was associated with motor deficits.

The $\alpha$-synuclein fibrils and ribbons could recruit and imprint their intrinsic structure to endogenous $\alpha$-synuclein. All forms of the protein were also found in the CNS after systemic administration, which is consistent with the hypothesis that synucleinopathies might not begin in the brain.

In a separate study, Dettmer and colleagues used chemical crosslinking to quantify physiological and pathogenic a-synuclein forms in human brain tissue from a patient undergoing resective surgery for epilepsy. Approximately 75\% of $\alpha$-synuclein was found to exist as tetramers in the cytosol, the rest being monomers.

Dettmer et al. observed a similar tetramer-monomer ratio in wild-type mice, but in three genetic mouse models of familial $\mathrm{PD}$, the tetramer-monomer ratio was consistently reduced by $20-30 \%$.

Moreover, in neurons derived from induced pluripotent stem cells from a patient with familial $\mathrm{PD}$, the investigators noted a $>25 \%$ shift from $a$-synuclein tetramers to monomers. When multiple mutations linked to PD were expressed in neurons, the strong shift from tetramers to free monomers led to frank neurotoxicity and a-synuclein-immunoreactive cytoplasmic inclusions.

Further investigations will be necessary to elucidate how a relative increase in cellular monomers encourages the formation of pathogenic oligomers, ribbons or fibrils. Nevertheless, these studies into the initiation of synucleinopathies represent crucial advances in the quest for early diagnosis and treatments.

\section{Alex Chase}

Original articles Peelaerts, W. et al. $\alpha$-Synuclein strains cause distinct synucleinopathies after local and systemic administration. Nature $\mathbf{5 2 2}$, 340-344 (2015) | Dettmer, U. et al. Parkinsoncausing $a$-synuclein missense mutations shift native tetramers to monomers as a mechanism for disease initiation. Nat. Commun. 6, 7314 (2015) 